Original Research Paper

\title{
Proportional Hazard Inverse Weibull Distribution and Associated Inference
}

\author{
${ }^{1}$ Suja M. Aboukhamseen, ${ }^{1}$ Mohamed E. Ghitany and ${ }^{2}$ Ramesh C. Gupta \\ ${ }^{I}$ Department of Statistics and Operations Research, \\ Faculty of Science, Kuwait University, P.O. Box 5969, Safat 13060, Kuwait \\ ${ }^{2}$ Department of Mathematics and Statistics, University of Maine, Orono, ME 04469-5752, USA
}

Article history

Received: 26-01-2016

Revised: 06-04-2016

Accepted: 15-04-2016

Corresoonding Author:

Suja M. Aboukhamseen

Department of Statistics and

Operations Research, Faculty

of Science, Kuwait University,

P.O. Box 5969, Safat 13060,

Kuwait

Email: saboukhamseen@yahoo.com

\begin{abstract}
In this study, we have considered the proportional hazard version of the inverse Weibull distribution. It has been shown that the density and hazard rate functions are unimodal and the mean residual life function is bathtub shaped. For the considered model, many functions of the parameters, such as the mean, variance, coefficient of variation and the critical points of the density, hazard rate and mean residual life functions cannot be given in explicit form. As a result, the variances of the maximum likelihood estimators of such functions cannot be given in explicit form to construct the usual asymptotic confidence interval. In this study, we use the percentile bootstrap estimation method to estimate such variances to construct the asymptotic confidence intervals. The asymptotic confidence intervals are compared with the bootstrap confidence intervals. Simulation studies are carried out to examine the performance of the maximum likelihood and bootstrap estimators. An example is provided to illustrate the procedure.
\end{abstract}

Keywords: Inverse Weibull Distribution, Moments, Probability Density Function, Hazard Rate Function, Mean Residual Life Function, Bootstrap Estimation

\section{Introduction}

The proportional hazard model has been widely used to analyze survival data. More specifically, let $Y$ be a random variable with survival function $S_{1}(y)$ and hazard rate function $\lambda_{1}(y)$. Then a random variable $X$ with survival function $S(x)=\left[S_{1}(x)\right]^{\alpha}, \alpha>0$, has proportional hazard rate function $\lambda(x)=\alpha \lambda_{1}(x)$. The parameter $\alpha$ is called a fraility parameter, see for example Marshall and Olkin (2007).

The inverse Weibull (IW) distribution with survival function given by

$$
S_{1}(y)=1-e^{-\lambda y^{-\beta}}, y>0, \beta, \lambda>0
$$

can be used to model a variety of failure characteristics such as infant mortality, useful life and wear out periods. For the structural properties of the IW distribution, we refer the reader Khan et al. (2008a; 2008b). The maximum likelihood and the least square estimations of the inverse Weibull distribution have been studied by Calabria and Pulcini (1990).
In various situations, it is more appropriate to employ the proportional hazard model than the original model. In this study, we are interested in the Proportional hazard Inverse Weibull (PHIW) distribution with survival function given by

$$
S(x)=\left(1-e^{-\lambda x^{-\beta}}\right)^{\alpha}, x>0, \alpha, \beta, \lambda>0 .
$$

Note that when $\alpha=1$, the PHIW distribution reduces to the IW distribution. Also, when $\beta=1$, the PHIW distribution reduces to the Generalized Inverted Exponential (GIE) distribution studied by Abouammoh and Alshingiti (2009).

It may be noted that analogues to the proportional hazard model, the proportional reversed hazard rate model has also been studied in the literature. In this case, the power of the distribution function is considered instead of the power of the survival function. For more information on the proportional reversed hazard rate model, we refer the reader to Gupta and Gupta (2007). 
The main purpose of this paper is to study the bootstrap estimation of the parameters of the PHIW distribution and the associated functions including the mean, variance, coefficient of variation, the probability density, the hazard rate and mean residual life functions. The paper is organized as follows: In section 2, we consider the shape of the density, hazard rate and mean residual life functions. The moments (including the negative moments) and the associated measures are given in section 3 . In section 4 , we study the maximum likelihood and bootstrap estimation of functions of the parameters. Simulation studies are carried out in section 5 to investigate the performance of the estimators of various functions of the parameters. An application is provided in section 6. Finally, some conclusion and comments are given.

\section{Probability Density, Hazard Rate and Mean Residual Life Functions}

\subsection{Probability Density Function}

The Probability Density Function (PDF) of the PHIW distribution, with survival function (2), is given by:

$$
f(x)=\alpha \beta \lambda\left(1-e^{-\lambda x^{-\beta}}\right)^{\alpha-1} x^{-\beta-1} e^{-\lambda x^{-\beta}}, x>0, \alpha, \beta, \lambda>0 .
$$

The following theorem investigates the shape of the PDF of the PHIW distribution.

\section{Theorem 1}

The PDF $f(x)$ is unimodal for all $\alpha, \beta, \lambda>0$, with $f(0)$ $=f(\infty)=0$.

\section{Proof.}

It is clear that $f(0)=f(\infty)=0$. The first derivative of $f(x)$ can be written in the form

$$
f^{\prime}(x)=\frac{f(x)}{x\left(1-e^{-\lambda x^{-\beta}}\right)} \phi(x)
$$

where,

$$
\begin{aligned}
& \phi(x)=[\beta+1-\alpha \beta y(x)] e^{-y(x)} \\
& +\beta y(x)-(\beta+1), y(x)=\lambda x^{-\beta} .
\end{aligned}
$$

Note that

$$
\phi^{\prime}(x)=-y^{\prime}(x) w(x)
$$

where

$$
w(x)=[\alpha \beta+\beta+1-\alpha \beta(x)] e^{-y(x)}-\beta .
$$

In the following, we show that $w(x)$ changes sign from negative to positive. Since

$$
w^{\prime}(x)=-y^{\prime}(x)[2 \alpha \beta+\beta+1-\alpha \beta y(x)] e^{-y(x)}
$$

it follows that $x_{0}=[\alpha \beta \lambda /(2 \alpha \beta+\beta+1)]^{1 / \beta}$ is a unique critical point where $w(x)$ has an absolute minimum, since

$$
w^{\prime \prime}\left(x_{0}\right)=\alpha \beta\left[y^{\prime}\left(x_{0}\right)\right]^{2} e^{-y\left(x_{0}\right)}>0 .
$$

Since $w(0)=-\beta$ and $w(\infty)=\alpha \beta+1$, it follows that $w(x)$ changes sign from negative to positive. This implies that $\phi^{\prime}(x)$ also changes sign from negative to positive, since $-y^{\prime}(x)>0$. Finally, since $\phi(0)=\infty$ and $\phi(\infty)=0$, $\phi(x)$ decreases from infinity to a negative value then increases to zero, i.e., $\phi(x)$ changes sign from positive to negative. That is, $f^{\prime}(x)$ changes sign from positive to negative proving the unimodality of $f(x)$.

\section{Remarks}

- The mode, $x_{f}$, is the solution of the non-linear equation $f^{\prime}\left(x_{f}\right)=0$ or equivalently

$$
\begin{aligned}
& \phi\left(x_{f}\right)=\left[\beta+1-\alpha \beta y\left(x_{f}\right)\right] e^{-y\left(x_{f}\right)} \\
& +\beta y\left(x_{f}\right)-(\beta+1)=0, y\left(x_{f}\right)=\lambda x_{f}^{-\beta}
\end{aligned}
$$

- For $\alpha=1, \beta, \lambda>0$, i.e., the IW distribution, the mode $x_{f}$ has an explicit closed form: $x_{f}=[\beta \lambda /(\beta+1)]^{1 / \beta}$, see Jiang et al. (2001).

- $\quad$ For $\alpha, \beta, \lambda>0$, since $\phi(0)=\infty$ and

$$
\begin{aligned}
& \phi\left(u_{1}\right)=\phi\left[(\lambda / \log (\alpha+1))^{1 / \beta}\right]= \\
& -\frac{1}{\alpha}\{\alpha+\beta[\alpha-\log (\alpha+1)]\}<0
\end{aligned}
$$

since $\log (\alpha+1)<\alpha$, for all $\alpha>0$, the search for the mode $x_{f}$ can be limited to the interval $\left(0, u_{1}\right)$. For example, see section $6, \alpha=16.391, \beta=3.098, \lambda=$ 44.632, $u_{1}=2.429$ and $x_{f}=2.321 \in\left(0, u_{1}\right)$.

Figure 1 shows the PDF of the PHIW distribution for selected values of the parameters.

\subsection{Hazard Rate Function}

The Hazard Rate Function (HRF) of the PHIW distribution, with survival function (2) and PDF (3), is given by 


$$
h(x)=\frac{f(x)}{S(x)}=\frac{\alpha \beta \lambda x^{-\beta-1} e^{-\lambda x^{-\beta}}}{1-e^{-\lambda x^{-\beta}}}, x>0, \alpha, \beta, \lambda>0 .
$$

The following theorem investigates the shape of the HRF of the PHIW distribution.

\section{Theorem 2}

The HRF $h(x)$ is unimodal (upside-down bathtub shape) for all $\alpha, \beta, \lambda>0$, with $h(0)=h(\infty)=0$.

\section{Proof.}

It is clear that $h(0)=h(\infty)=0$. Since $h(x)=\alpha h_{1}(x)$ where

$$
h_{i}(x)=\frac{\beta \lambda x^{-\beta-1} e^{-\lambda x^{-\beta}}}{1-e^{-\lambda x^{-\beta}}}, x>0, \beta, \lambda>0,
$$

is the HRF of the IW distribution with PDF

$$
f_{1}(x)=\beta \lambda x^{-\beta-1} e^{-\lambda x^{-\beta}}, x>0, \beta, \lambda>0
$$

and survival function $S_{1}(x)$. Clearly, the shape of $h(x)$ is the same of $h_{1}(x)$. Now, we use Glaser (1980) eta function of $f_{1}(x)$ :

$$
\eta(x)=-\left(\log f_{1}(x)\right)^{\prime}=(\beta+1) x^{-1}-\beta \lambda x^{-\beta-1}
$$

to determine the shape of $h_{1}(x)$. Since

$$
\eta^{\prime}(x)=(\beta+1) x^{-\beta-2}\left(\beta \lambda-x^{\beta}\right)
$$

it follows that $u_{2}=(\beta \lambda)^{1 / \beta}$ is the unique critical point which maximizes $\eta(x)$, since

$$
\eta^{\prime \prime}\left(u_{2}\right)=-(\beta+1) \beta^{2} \lambda u_{2}^{-\beta-3}<0
$$

That is, $\eta(x)$ is unimodal with $\eta(0)=-\infty$ and $\eta(\infty)=$ 0 . Finally, since $f_{1}(0)=0$, it follows that $h_{1}(x)$ and hence $h(x)$, is also unimodal.

\section{Remarks}

The critical point, $x_{h}$, of $h(x)$ is the unique solution of $h^{\prime}\left(x_{h}\right)=0$, which is equivalent to solving the non-linear equation

$$
\begin{aligned}
& \psi\left(x_{h}\right)=(\beta+1) e^{-y\left(x_{h}\right)}+\beta y\left(x_{h}\right) \\
& -(\beta+1)=0, y\left(x_{h}\right)=\lambda x_{h}^{-\beta}
\end{aligned}
$$

which is independent of $\alpha$.

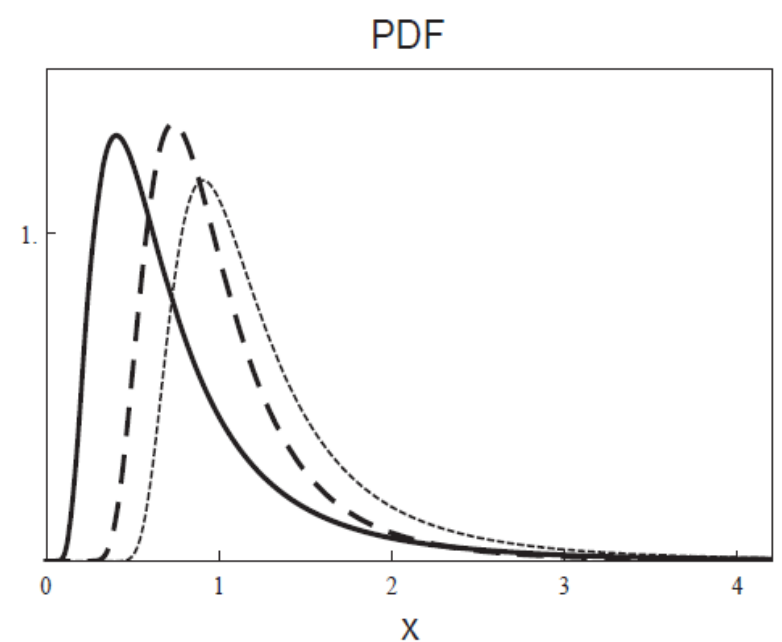

Fig. 1. PDF of the PHIW distribution for $(\alpha, \beta, \lambda)$ : $(3,1,1)$ (solid), $(2,2,1)$ (dashed) and $(1,3,1)$ (dotted)

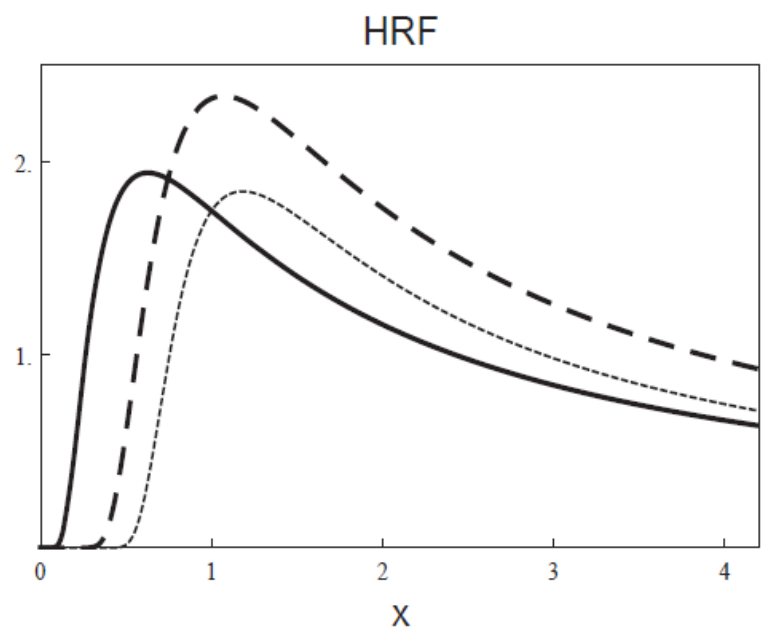

Fig. 2. HRF of the PHIW distribution for $(\alpha, \beta, \lambda)$ : $(3,1,1)$ (solid), $(2,2,1)$ (dashed) and $(1,3,1)$ (dotted)

It is clear that $\psi(0)=\infty$ and $\psi\left(u_{2}\right)=\psi\left[(\beta \lambda)^{1 / \beta}\right]=-\beta$ $\left[1-(1+1 / \beta) e^{-1 / \beta}\right]<0$, since $(1+z) e^{-z}<1$, for all $z>0$. This will be useful in finding the unique root $x_{h}$ of $\psi\left(x_{h}\right)$ by limiting the search on the interval $\left(0, u_{2}\right)$. For example, see section $6, \alpha=16.391, \beta=3.098, \lambda=$ 44.632, $u_{2}=4.909$ and $x_{h}=4.045 \in\left(0, u_{2}\right)$.

Figure 2 shows the HRF of the PHIW distribution for selected values of the parameters.

\subsection{Mean Residual Life Function}

Using the substitution $y=\lambda x^{-\beta}$, the mean of the PHIW distribution is given by

$$
\mu=\int_{0}^{\infty} S(x) d x=\frac{\lambda^{1 / \beta}}{\beta} \int_{0}^{\infty} y^{-1 / \beta-1}\left(1-e^{-y}\right)^{\alpha} d y
$$


For $0<\alpha \beta \leq 1$, using the inequality $1-e^{-y} \geq y e^{-y}$, for all $y \geq 0$, we have

$$
\mu \geq \frac{\lambda^{1 / \beta}}{\beta} \int_{0}^{\infty} y^{(\alpha \beta-1) \beta-1} e^{-\alpha y} d y=\infty .
$$

For $\alpha \beta>1$, using the inequalities $1-e^{-y} \leq y$ and $1-$ $e^{-y} \leq 1$, for all $y \geq 0$, respectively, we have

$$
\begin{aligned}
& \mu \geq \frac{\lambda^{1 / \beta}}{\beta}\left\{\int_{0}^{1} y^{-1 / \beta-1}\left(1-e^{-y}\right)^{\alpha} d y+\int_{1}^{\infty} y^{-1 / \beta-1}\left(1-e^{-y}\right)^{\alpha} d y\right\} \\
& \leq \frac{\lambda^{1 / \beta}}{\beta}\left\{\int_{0}^{1} y^{(\alpha \beta-1) / \beta-1} d y+\int_{1}^{\infty} y^{-1 / \beta-1} d y\right\} \\
& =\frac{\alpha \beta \lambda^{1 / \beta}}{\alpha \beta-1}<\infty .
\end{aligned}
$$

For all $x>0$, the Mean Residual Life Function (MRLF) of the PHIW distribution, with survival function (2) and PDF (3), is given by

$$
\begin{aligned}
& \mu(x)=E(X-x \mid X>x) \\
& =\frac{1}{S(x)} \int_{x}^{\infty} y f(y) d y-x \\
& =\frac{\alpha \beta \lambda}{\left(1-e^{-\lambda x-\beta}\right)^{\alpha}} \int_{x}^{\infty}\left(1-e^{-\lambda y-\beta}\right)^{\alpha-1} y^{-\beta} e^{-\lambda y-\beta} d y-x .
\end{aligned}
$$

Note that $\mu(0)=\mu<\infty$, provided $\alpha \beta>1$ and $\mu(\infty)=$ $1 / h(\infty)=\infty$.

For $\alpha=1,2,3, \ldots$ and $\beta>1$, using the substitution $z$ $=\lambda y^{-\beta}$, we have

$$
\begin{aligned}
& \mu(x)=\frac{\alpha \lambda^{1 / \beta}}{\left(1-e^{-\lambda x-\beta}\right)^{\alpha}} \int_{0}^{\lambda x^{-\beta}}\left(1-e^{-z}\right)^{\alpha-1} z^{-1 / \beta} e^{-z} d z-x \\
& =\frac{\alpha \lambda^{1 / \beta}}{\left(1-e^{-\lambda x-\beta}\right)^{\alpha}} \sum_{i=0}^{\alpha-1}\left(\begin{array}{c}
\alpha-1 \\
i
\end{array}\right)(-1)^{i} \int_{0}^{\lambda x^{-\beta}} z^{-1 / \beta} e^{-(i+1) z} d z-x \\
& =\frac{\alpha \lambda^{1 / \beta}}{\left(1-e^{-\lambda x-\beta}\right)^{\alpha}} \sum_{i=0}^{\alpha-1}\left(\begin{array}{c}
\alpha-1 \\
i
\end{array}\right) \frac{(-1)^{i}}{(i+1)^{1-1 / \beta}} \gamma(1-1 / \beta,(i+1) \lambda x-\beta)-x .
\end{aligned}
$$

where, $\gamma(a, t)=\int_{0}^{t} u^{a-1} e^{-u} d u, a>0$, is the lower incomplete gamma function for which $\gamma(a, 0)=0$ and $\gamma(a, \infty)=\Gamma(a)$ is the gamma function. have

For the special case, $\alpha=1$, i.e., IW distribution, we

$$
\mu(x)=\frac{\lambda^{1 / \beta} \gamma\left(1-1 / \beta, \lambda x^{-\beta}\right)}{1-e-\lambda x^{-\beta}}-x, \beta>1 .
$$

Before we determine the shape of $\mu(x)$, we need the following lemma.

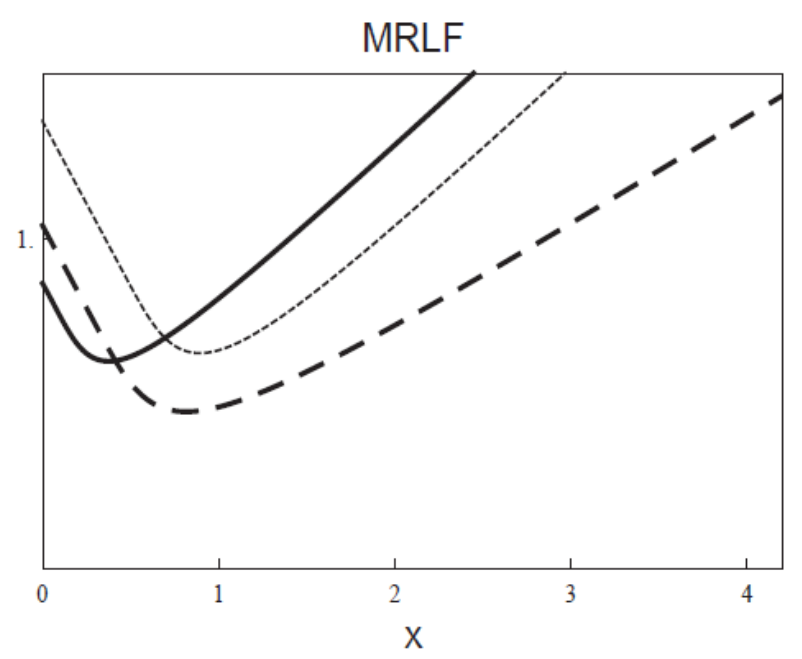

Fig. 3. MRLF of the PHIW distribution for $(\alpha, \beta, \lambda):(3,1,1)$ (solid), $(2,2,1)$ (dashed) and $(1,3,1)$ (dotted)

\section{Lemma 1}

Gupta and Akman (1995) If $h(x)$ is upside-down bathtub shape with a unique critical point $x_{h}$ and $f(0) \mu$ $<1$, then $\mu(x)$ is bathtub shape with a unique critical point $x_{m}$. The critical point $x_{m}$ is the solution of the non-linear equation

$h\left(x_{m}\right) \mu\left(x_{m}\right)-1=0$.

Moreover, $x_{m}<x_{h}$.

Using the condition $\alpha \beta>1$ and Lemma 1, we are now able to determine the shape of the MRLF of the PHIW distribution as follows.

\section{Theorem 3}

The MRLF $\mu(x)$ is upside-down bathtub shape with unique critical point $x_{m}$ for all $\alpha, \beta>0$ such that $\alpha \beta>1$ and $\lambda>0$.

Proof.

Since $\alpha \beta>1$ implies that $\mu<\infty$ and $f(0)=0$, we have $f(0) \mu=0<1$. Therefore, using Lemma 1 , the MRLF is bathtub shaped.

Figure 3 shows the MRLF of the PHIW distribution for selected values of the parameters.

\section{Moments and Associated Measures}

The $k$ th raw moment of the PHIW distribution is given by

$$
\mu_{k}^{\prime}=\int_{0}^{\infty} k x^{k-1} S(x) d x=\frac{k \lambda^{k / \beta}}{\beta} \int_{0}^{\infty} y^{-k / \beta-1}\left(1-e^{-y}\right)^{\alpha} d y .
$$


By exactly the same steps as in subsection 2.3, $\mu_{k}^{\prime}$ exists only when $\alpha \beta>k$. For positive real values $\alpha$ and $\beta$, the moments cannot be expressed in closed form expressions. However, for $\alpha=1,2,3, \ldots$, we have

$$
\mu_{k}^{\prime}=\alpha \lambda^{k / \beta} \Gamma(1-k / \beta) \sum_{i=0}^{\alpha-1}\left(\begin{array}{c}
\alpha-1 \\
i
\end{array}\right) \frac{(-1)^{i}}{(i+1)^{1-k / \beta}}, \beta>k .
$$

For the special case, $\alpha=1$, i.e., IW distribution, we have:

$$
\mu_{k}^{\prime}=\lambda^{k / \beta} \Gamma(1-k / \beta), \beta>k
$$

In this case, the mean, variance and coefficient of variation, respectively, are

$$
\begin{aligned}
& \mu=\lambda^{1 / \beta} \Gamma(1-1 / \beta), \beta>1, \\
& \sigma^{2}=\lambda^{2 / \beta}\left[\Gamma(1-2 / \beta)-\Gamma^{2}(1-1 / \beta)\right], \beta>2, \\
& c v=\frac{\sqrt{\Gamma(1-2 / \beta)-\Gamma^{2}(1-1 / \beta)}}{\Gamma(1-1 / \beta)}, \beta>2 .
\end{aligned}
$$

Note that if $X \sim \operatorname{PHIW}(\alpha, \beta, \gamma)$, then $Z=1 / X$ has the exponentiated Weibull distribution (Mudholkar and Srivastava 1993) with cumulative distribution function

$$
F_{Z}(z)=\left(1-e^{-\lambda z^{\beta}}\right)^{\alpha}, z>0, \alpha, \beta, \gamma>0
$$

Now the negative moments of the PHIW distribution are given by

$$
\begin{aligned}
& E\left(X^{-k}\right)=E\left(Z^{k}\right) \\
& =\alpha \lambda^{-k / \beta} \Gamma(k / \beta+1) \sum_{i=0}^{\infty} \frac{(1-\alpha)}{i !(i+1)^{k / \beta+1}}
\end{aligned}
$$

where, for any real number $c$ :

$$
(c)_{i}= \begin{cases}1, & \text { if } i=0 \\ c(c+1) \ldots(c+i-1), & \text { if } i \geq 1\end{cases}
$$

is the Pochhammer symbol, for more details see Choudhury (2005).

For $\alpha=1,2, \ldots$, we have:

$$
E\left(X^{-k}\right)=\alpha \lambda^{-k / \beta} \Gamma(k / \beta+1) \sum_{i=0}^{\alpha-1} \frac{(1-\alpha)_{i}}{i !(i+1)^{k / \beta+1}} .
$$
have

In particular, if $\alpha=1$, i.e., the IW distribution, we

$$
E\left(X^{-k}\right)=\lambda^{-k / \beta} \Gamma(k / \beta+1) .
$$

\section{Maximum Likelihood Estimation}

Let $x_{1}, x_{2}, \ldots, x_{n}$ be a random sample of size $n$ from the PHIW distribution with PDF (3). The log-likelihood function is given by

$$
\begin{aligned}
& \ell_{n}(\theta)=\sum_{i=1}^{n} \log f\left(x_{i}\right), \\
& =n \log (\alpha \beta \lambda)-\sum_{i=1}^{n}\left\{\begin{array}{l}
(\beta+1) \log \left(x_{i}\right) \\
+\lambda x_{i}^{-\beta}-(\alpha-1) \log \left(1-e^{-\lambda x_{i}^{-\beta}}\right)
\end{array}\right\} .
\end{aligned}
$$

The maximum likelihood estimates (MLEs) $(\hat{\alpha}, \hat{\beta}, \hat{\lambda})$ of the parameters $(\alpha, \beta, \lambda)$ are the solution of the system of non-linear equations

$\frac{\partial \ell_{n}(\theta)}{\partial \alpha}=\frac{n}{\alpha}+\sum_{i=1}^{n} \log \left(1-e^{-\lambda x_{i}^{-\beta}}\right)=0$,

$\frac{\partial \ell_{n}(\theta)}{\partial \beta}=\frac{n}{\beta}+\sum_{i=1}^{n}\left\{1-\lambda x_{i}^{-\beta}+\frac{(\alpha-1) \lambda x_{i}^{-\beta}}{e^{\lambda x_{i}^{-\beta}}-1}\right\} \log \left(x_{i}\right)=0$,

$\frac{\partial \ell_{n}(\theta)}{\partial \lambda}=\frac{n}{\lambda}+\sum_{i=1}^{n}\left\{-1 \frac{(\alpha-1)}{e^{\lambda x_{i}^{-\beta}}-1}\right\} \log \left(x_{i}^{-\beta}\right)=0$.

Note that (8) implies that

$$
\hat{\alpha}=\frac{-n}{\sum_{i=1}^{n} \log \left(1-e^{-\hat{\lambda} x_{i}^{-\hat{\beta}}}\right)}=\xi(\hat{\beta}, \hat{\lambda}) .
$$

Hence, the 3 -dimensional maximization in $(\alpha, \beta, \lambda)$ is reduced to 2 -dimensional maximization in $(\beta, \lambda)$ :

$\frac{n}{\beta}-\sum_{i=1}^{n}\left\{1-\lambda x_{i}^{-\beta}+\frac{[\xi(\beta, \lambda)-1] \lambda x_{i}^{-\beta}}{e^{\lambda x_{i}^{-\beta}}-1}\right\} \log \left(x_{i}\right)=0$,

$\frac{n}{\lambda}-\sum_{i=1}^{n}\left\{1-\frac{\xi(\beta, \lambda)-1}{e^{\lambda x_{i}^{-\beta}}-1}\right\} x_{i}^{-\beta}=0$.

The solutions of the last two nonlinear equations provide the MLEs $\hat{\beta}$ and $\hat{\lambda}$ of the parameters $\beta$ and $\lambda$, from which the MLE $\hat{\alpha}=\xi(\hat{\beta}, \hat{\lambda})$ of the parameter $\alpha$ is obtained.

The observed information matrix is

$$
I_{n}(\theta)=\left[-\frac{\partial^{2} \ell_{n}(\theta)}{\partial \theta_{i} \partial \theta_{j}}\right]=\left[\begin{array}{ccc}
I_{n}^{11}(\theta) & I_{n}^{12}(\theta) & I_{n}^{13}(\theta) \\
I_{n}^{12}(\theta) & I_{n}^{22}(\theta) & I_{n}^{23}(\theta) \\
I_{n}^{12}(\theta) & I_{n}^{23}(\theta) & I_{n}^{33}(\theta)
\end{array}\right]
$$


where

$$
\begin{aligned}
& I_{n}^{11}(\theta)=-\frac{\partial^{2} \ell_{n}(\theta)}{\partial \alpha^{2}}=\frac{n}{\alpha^{2}}, \\
& I_{n}^{22}(\theta)=-\frac{\partial^{2} \ell_{n}(\theta)}{\partial \beta^{2}}=\frac{n}{\beta^{2}} \\
& +\lambda \sum_{i=1}^{n}\left\{1+(\alpha-1) \frac{1-\left(1-\lambda x_{i}^{-\beta}\right) e^{\lambda x_{i}^{-\beta}}}{\left(e^{\lambda x_{i}^{-\beta}}-1\right)^{2}}\right\} x_{i}^{-\beta}\left[\log \left(x_{i}\right)\right]^{2}, \\
& I_{n}^{33}(\theta)=-\frac{\partial^{2} \ell_{n}(\theta)}{\partial \lambda^{2}}=\frac{n}{\lambda^{2}}-(\alpha-1) \sum_{i=1}^{n} \frac{x_{i}^{-2 \beta} e^{\lambda x_{i}^{-\beta}}}{\left(e^{\lambda x_{i}^{-\beta}}-1\right)^{2}}, \\
& I_{n}^{12}(\theta)=-\frac{\partial^{2} \ell_{n}(\theta)}{\partial \alpha \partial \beta}=\lambda \sum_{i=1}^{n} \frac{x_{i}^{-\beta} \log \left(x_{i}\right)}{e^{\lambda x_{i}^{-\beta}}-1}, \\
& I_{n}^{13}(\theta)=-\frac{\partial^{2} \ell_{n}(\theta)}{\partial \alpha \partial \lambda}=-\sum_{i=1}^{n} \frac{x_{i}^{-\beta}}{e^{\lambda x_{i}^{-\beta}}-1}, \\
& -\sum_{i=1}^{23}\left\{1+(\theta)=-\frac{\partial^{2} \ell_{n}(\theta)}{\partial \beta \partial \lambda}=(\alpha-1) \frac{1-\left(1-\lambda x_{i}^{-\beta}\right) e^{\lambda x_{i}^{-\beta}}}{\left(e \lambda x_{i}^{-\beta}-1\right)^{2}}\right\} x_{i}^{-\beta} \log \left(x_{i}\right) .
\end{aligned}
$$

Under fairly mild regularity conditions, see Lehmann and Casella (1998), pp. 461-463, we have the following result

$$
\hat{\theta} \underset{\sim}{d} N_{3}\left(\theta, I_{n}^{-1}(\hat{\theta})\right)
$$

where, $\underset{\sim}{d}$ means approximately distributed and $N_{3}(.,$. denotes the trivariate normal distribution.

For a real-valued function of the parameters $\tau(\theta)$, we have

$$
\tau(\hat{\theta}) \underset{\sim}{d} N(\tau(\theta), \operatorname{var}[\tau(\hat{\theta})]) .
$$

The asymptotic $100(1-\alpha) \%$ confidence interval for $\tau$ $(\theta)$ is given by

$$
\tau(\hat{\theta}) \pm z_{\alpha / 2} \sqrt{\widehat{\operatorname{var}}[\tau(\hat{\theta})]}
$$

where, $z_{\alpha / 2}$ is the upper $\alpha / 2$ percentile of the standard normal distribution.

When the function $\tau(\theta)$ has no explicit expression in the parameters, as the case in this study, then $\operatorname{var}[\tau(\hat{\theta})]$ has also no explicit expression and is usually estimated using simulation-based methods such as percentile bootstrap, see for example Davison and Hinkley (1997), where $B$ random samples are selected with replacement from the given data set. For the $j$ th bootstrap sample, the
$M L E \hat{\theta}_{j}^{*}$ of $\theta$ and the $M L E \hat{\tau}_{j}^{*}=\tau\left(\hat{\theta}_{j}^{*}\right)$ of $\tau(\theta)$ are calculated. The mean and variance of all bootstrap estimates $\hat{\tau}_{1}^{*}, \hat{\tau}_{2}^{*}, \ldots, \hat{\tau}_{B}^{*}$, respectively, are given by

$$
\begin{aligned}
& \bar{\tau}^{*}=\frac{1}{B} \sum_{j=1}^{B} \hat{\tau}_{j}^{*}, \\
& s^{* 2}=\frac{1}{B-1} \sum_{j=1}^{B}\left(\hat{\tau}_{j}^{*}-\bar{\tau}^{*}\right)^{2} .
\end{aligned}
$$

Now, we can use $\bar{\tau}^{*}$ and $s^{* 2}$ as estimates of $\tau(\theta)$ and $\operatorname{var}[\tau(\hat{\theta})]$, respectively. Moreover, in this case, the following confidence intervals can be constructed.

The asymptotic $100(1-\alpha) \%$ confidence interval for $\tau$ $(\theta)$ is given by

$$
\tau(\hat{\theta}) \pm z_{\alpha / 2} s^{*}
$$

The $100(1-\alpha) \%$ percentile bootstrap confidence interval for $\tau(\theta)$ is given by

$$
\left(\hat{\tau}^{*(\alpha / 2)}, \hat{\tau}^{*(1-\alpha / 2)}\right)
$$

where, $\hat{\tau}^{*(p)}$ is the $p$ th percentile of the bootstrap estimates $\hat{\tau}_{1}^{*}, \hat{\tau}_{2}^{*}, \ldots, \hat{\tau}_{B}^{*}$.

\section{Simulation Studies}

Simulation of an observation $x$ from the PHIW distribution is obtained by solving the equation $S(x)=1$ $u$ where $u$ is an observation from the uniform distribution over $(0,1)$. It follows that

$$
x=\left\{\frac{-\lambda}{\log \left[1-(1-u)^{1 / \alpha}\right]}\right\}^{1 / \beta} .
$$

\section{Design of the Simulation Studies}

- Sample sizes: $n=50,100$

- Population parameters: $(\alpha, \beta, \lambda)=(10,5,1),(5,10$, $1)$. Table 1 shows the true values of the parameters used in the simulations

- Number of bootstrap samples: $B=500$

- Number of simulation runs $N=500$

\section{Computations}

- All computations in the simulation studies were done using the $\mathrm{R}$ package Version 3.1.2, see RDCT (2014)

- Maximum likelihood estimates of the parameters were obtained using the $\mathrm{R}$ general purpose 
optimization function optim which implements the Nelder-Mead technique

- The bootstrap samples were obtained using the $\mathrm{R}$ package boot by Canty and Ripley (2015)

\section{Simulation Criteria}

Let $\tilde{\tau} \equiv \tau(\tilde{\theta})$ be the MLE or bootstrap estimate of $\tau \equiv$ $\tau(\theta)$ and $\tilde{\tau}_{[i]} \equiv \tau\left(\tilde{\theta}_{[i]}\right)$ be the estimate of $\tau$ in the $i^{\text {th }}$ simulation run, $i=1,2, \ldots, N$.

The following criteria are used to assess the performance of the MLEs/bootstrap estimates of $\tau \equiv \tau(\theta)$ and associated confidence intervals.

- Bias:

$$
\operatorname{Bias}(\tilde{\tau})=\frac{1}{N} \sum_{i=1}^{N}\left(\tilde{\tau}_{[i]}-\tau\right)
$$

- $\quad$ Mean-square error (MSE):

$$
\operatorname{MSE}(\tilde{\tau})=\frac{1}{N} \sum_{i=1}^{N}\left(\tilde{\tau}_{[i]}-\tau\right)^{2}
$$

- Coverage probability (CP): Percentage of the true value of the parameter which falls in a given confidence interval

- Confidence interval width: The range of the confidence interval

\section{Analysis of the Simulation Studies}

- For the mean, variance and coefficient of variation (Table 2), both confidence intervals are performing almost the same in terms of the coverage probability as well as their width

- In the case of the estimation of critical points of the PDF, HRF and MRLF (Table 3), the coverage probability of both confidence intervals is the same while the bootstrap intervals are outperforming the asymptotic intervals in terms of their width

Table 1. True values of the functions of the parameters used in the simulations

\begin{tabular}{lllllll}
\hline$(\alpha, \beta, \lambda)$ & $\mu$ & $\sigma^{2}$ & $c v$ & $x_{f}$ & $x_{h}$ & $x_{m}$ \\
\hline$(5,10,1)$ & 0.9657 & 0.0025 & 0.0539 & 0.9243 & 1.1784 & 1.1555 \\
$(10,5,1)$ & 0.8232 & 0.0047 & 0.0832 & 0.8130 & 1.2158 & 1.1888 \\
\hline
\end{tabular}

\begin{tabular}{|c|c|c|c|c|c|c|}
\hline$(\alpha, \beta, \lambda)$ & $n$ & Method & Bias & MSE & $\mathrm{CP}(\%)$ & C.I. width \\
\hline \multicolumn{7}{|l|}{ Mean } \\
\hline \multirow[t]{4}{*}{$(5,10,1)$} & 50 & Asymptotic & 0.0001 & 0.0000 & 95.40 & 0.0275 \\
\hline & & Bootstrap & 0.0001 & 0.0000 & 94.80 & 0.0277 \\
\hline & 100 & Asymptotic & -0.0001 & 0.0000 & 95.20 & 0.0196 \\
\hline & & Bootstrap & -0.0001 & 0.0000 & 95.00 & 0.0198 \\
\hline \multirow[t]{4}{*}{$(10,5,1)$} & 50 & Asymptotic & 0.0006 & 0.0001 & 93.40 & 0.0373 \\
\hline & & Bootstrap & 0.0006 & 0.0001 & 92.80 & 0.0376 \\
\hline & 100 & Asymptotic & 0.0002 & 0.0000 & 95.80 & 0.0268 \\
\hline & & Bootstrap & 0.0003 & 0.0000 & 95.80 & 0.0270 \\
\hline \multicolumn{7}{|l|}{ Variance } \\
\hline \multirow[t]{4}{*}{$(5,10,1)$} & 50 & Asymptotic & -0.0001 & 0.0000 & 88.40 & 0.0022 \\
\hline & & Bootstrap & -0.0001 & 0.0000 & 89.20 & 0.0022 \\
\hline & 100 & Asymptotic & -0.0001 & 0.0000 & 92.20 & 0.0016 \\
\hline & & Bootstrap & -0.0001 & 0.0000 & 92.40 & 0.0016 \\
\hline \multirow[t]{4}{*}{$(10,5,1)$} & 50 & Asymptotic & -0.0001 & 0.0000 & 89.20 & 0.0040 \\
\hline & & Bootstrap & -0.0001 & 0.0000 & 89.40 & 0.0040 \\
\hline & 100 & Asymptotic & 0.0000 & 0.0000 & 94.00 & 0.0028 \\
\hline & & Bootstrap & 0.0000 & 0.0000 & 94.00 & 0.0028 \\
\hline \multicolumn{7}{|c|}{ Coefficient of variation } \\
\hline \multirow[t]{4}{*}{$(5,10,1)$} & 50 & Asymptotic & -0.0007 & 0.0000 & 91.00 & 0.0231 \\
\hline & & Bootstrap & -0.0011 & 0.0000 & 89.40 & 0.0233 \\
\hline & 100 & Asymptotic & -0.0004 & 0.0000 & 92.80 & 0.0165 \\
\hline & & Bootstrap & -0.0006 & 0.0000 & 92.80 & 0.0166 \\
\hline \multirow[t]{4}{*}{$(10,5,1)$} & 50 & Asymptotic & -0.0013 & 0.0001 & 93.40 & 0.0340 \\
\hline & & Bootstrap & -0.0020 & 0.0000 & 92.40 & 0.0351 \\
\hline & 100 & Asymptotic & -0.0002 & 0.0000 & 94.80 & 0.0242 \\
\hline & & Bootstrap & -0.0006 & 0.0000 & 94.00 & 0.0244 \\
\hline
\end{tabular}

Table 2. Simulation results for the estimation of mean, variance and coefficient of variation 
Table 3. Simulation results for the estimation of critical points of PDF, HRF and MRLF

\begin{tabular}{|c|c|c|c|c|c|c|}
\hline$(\alpha, \beta, \lambda)$ & $n$ & Method & Bias & MSE & $\mathrm{CP}(\%)$ & C.I. width \\
\hline \multicolumn{7}{|c|}{ Critical point of PDF } \\
\hline \multirow{3}{*}{$(5,10,1)$} & & Bootstrap & 0.1403 & 0.0517 & 97.20 & 1.6196 \\
\hline & 100 & Asymptotic & 0.0167 & 0.0662 & 96.20 & 1.0865 \\
\hline & & Bootstrap & 0.0545 & 0.0174 & 95.40 & 0.6484 \\
\hline \multirow[t]{4}{*}{$(10,5,1)$} & 50 & Asymptotic & 0.0097 & 0.0351 & 93.00 & 0.4479 \\
\hline & & Bootstrap & 0.0137 & 0.0008 & 94.80 & 0.1729 \\
\hline & 100 & Asymptotic & 0.0005 & 0.0001 & 95.20 & 0.0971 \\
\hline & & Bootstrap & 0.0020 & 0.0001 & 96.00 & 0.0536 \\
\hline \multicolumn{7}{|c|}{ Critical point of $\mathrm{HRF}$} \\
\hline \multirow[t]{4}{*}{$(5,10,1)$} & 50 & Asymptotic & 0.1153 & 0.2108 & 95.60 & 2.2538 \\
\hline & & Bootstrap & 0.2312 & 0.0731 & 96.00 & 2.2134 \\
\hline & 100 & Asymptotic & 0.0414 & 0.0724 & 99.00 & 1.5893 \\
\hline & & Bootstrap & 0.1183 & 0.0264 & 97.20 & 1.2926 \\
\hline \multirow[t]{4}{*}{$(10,5,1)$} & 50 & Asymptotic & 0.1325 & 0.1887 & 95.80 & 1.8136 \\
\hline & & Bootstrap & 0.2718 & 0.1447 & 97.80 & 1.6402 \\
\hline & 100 & Asymptotic & 0.0562 & 0.0514 & 98.60 & 1.0586 \\
\hline & & Bootstrap & 0.1104 & 0.0616 & 95.40 & 0.9683 \\
\hline \multicolumn{7}{|c|}{ Critical point of MRLF } \\
\hline \multirow[t]{4}{*}{$(5,10,1)$} & 50 & Asymptotic & 0.1518 & 0.3011 & 96.20 & 2.8704 \\
\hline & & Bootstrap & 0.3851 & 0.2172 & 96.40 & 2.7247 \\
\hline & 100 & Asymptotic & 0.0642 & 0.1435 & 98.80 & 1.9561 \\
\hline & & Bootstrap & 0.1859 & 0.0909 & 97.00 & 1.6775 \\
\hline \multirow[t]{4}{*}{$(10,5,1)$} & 50 & Asymptotic & 0.0884 & 0.1622 & 97.20 & 2.0358 \\
\hline & & Bootstrap & 0.2055 & 0.0920 & 98.00 & 1.9357 \\
\hline & 100 & Asymptotic & 0.0472 & 0.0440 & 99.20 & 1.0277 \\
\hline & & Bootstrap & 0.0803 & 0.0365 & 96.40 & 0.8916 \\
\hline
\end{tabular}

Thus, overall, the simulation studies indicate that both kind of confidence intervals are performing similar in terms of the coverage probabilities as well as their width.

Figure 4 shows approximately normal distribution for the sampling distribution of the MLEs of the mean, variance and coefficient of variation, indicating that the corresponding constructed asymptotic confidence intervals are appropriate. On the other hand, Figure 5 shows skewed sampling distribution of the MLEs of the critical point of the PDF, HRF and MRLF, indicating that the constructed asymptotic confidence intervals are inappropriate.

\section{Illustrative Example}

In this section, we present an illustrative example based on a real-life data set representing 98 measurements of the annual maxima of river flows $\left(\mathrm{m}^{3} \mathrm{~s}^{-1}\right)$ from 1900 to 1997 at BSWN station in Shunde area, Pearl River basin, China (Shao et al., 2004):

$1.678,2.285,2.371,2.255,2.275,2.14,2.464$, $2.052,2.542,2.355,1.957,2.32,2.487,2.358$, $2.567,3.123,2.052,2.464,2.566,2.438$, $2.438,2.285,2.409,2.499,2.595,2.076$, $2.404,2.261,2.267,2.189,2.362,2.675$,
$2.435,2.438,2.261,2.552,2.438,2.567,2.07$, 2.288, 2.46, 2.303, 2.512, 2.33, 2.729, 2.736, 2.381, 2.762, 2.249, 2.862, 2.376, 2.174, 1.89, $1.93,2.16,1.92,2.05,2.03,1.91,2.62,1.95$ $2.34,2.67,2.14,2.24,2.4,2.63,2.25,2.8$, $2.26,2.58,2.22,2.09,2.46,2.96,2.47,2.7$, $2.32,2.76,2.3,1.95,2.33,2.13,2.54,2.01$, $2.06,2.3,1.98,2.34,2.43,1.91,2.44,2.29$, $2.53,3.21,2.27,2.46,2.77$.

A summary of the basic descriptive sample statistics of this data set is given in Table 4 .

Table 5 shows the MLEs of the parameters, the estimated log-likelihood, Kolomogrov-Smirnov and Anderson-Darling goodness-of-fit tests of the fitted PHIW model. Both tests indicate that the fitted PHIW model is suitable for the given data set. This conclusion is also supported by the diagnostic plots presented in Fig. 6.

Figure 7 shows the location of the percentile bootstrap estimates of the critical points of the PDF, HRF and MRLF of the fitted PHIW model. The histograms of the percentile bootstrap estimates of the mean, variance, coefficient of variation and the critical points of the PDF, HRF and MRLF are presented in Fig. 8 which indicates that the histograms of the critical points are heavily skewed to the right. 
Mean

$$
\alpha=5, \beta=10, \lambda=1
$$

$$
\mathrm{n}=50
$$

$\mathrm{n}=100$

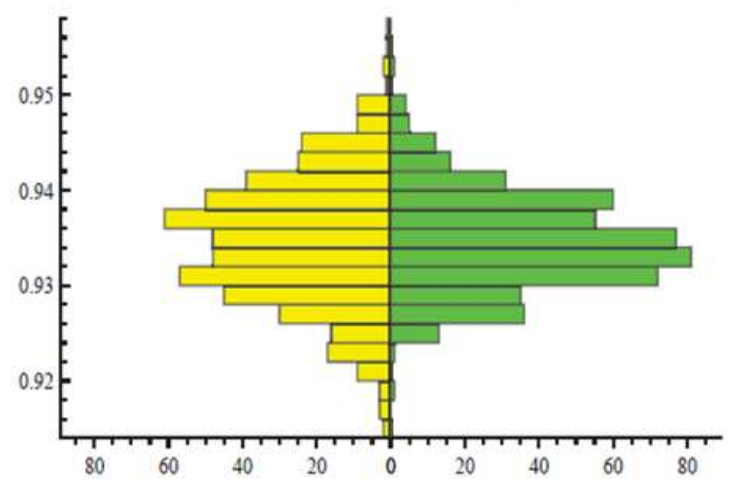

Variance

Variance

$\alpha=5, \beta=10, \lambda=1$

$$
\mathrm{n}=50
$$

$\mathrm{n}=100$

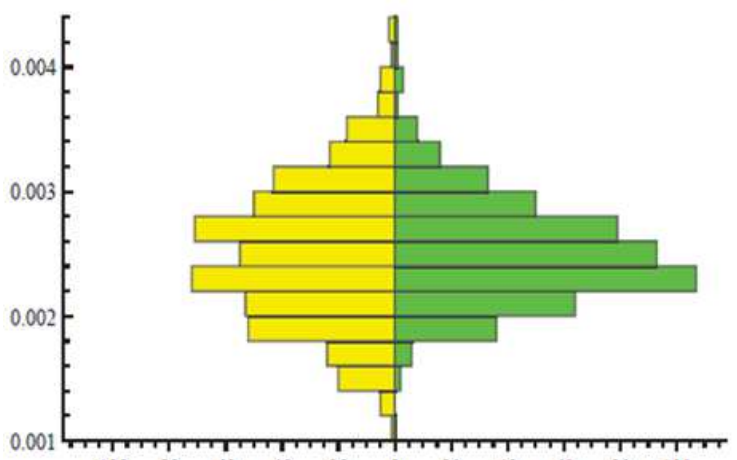

$\begin{array}{lllllllllll}100 & 80 & 60 & 40 & 20 & 0 & 20 & 40 & 60 & 80 & 100\end{array}$

Coefficient of variation

$$
\alpha=5, \beta=10, \lambda=1
$$

$$
\mathrm{n}=50
$$

$$
\mathrm{n}=100
$$

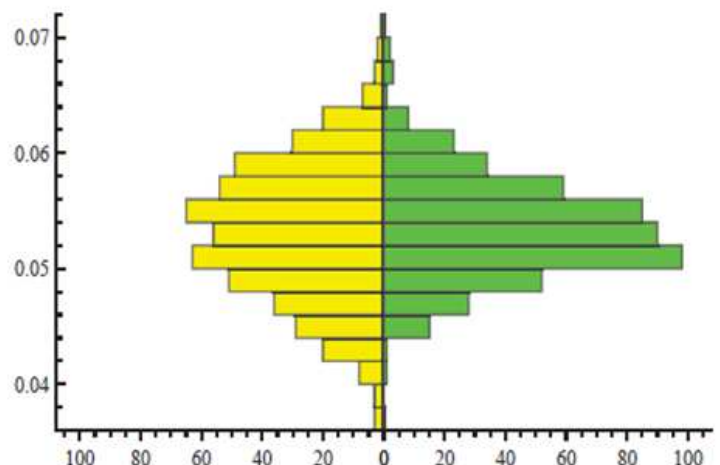

Mean

$\alpha=10, \beta=5, \lambda=1$

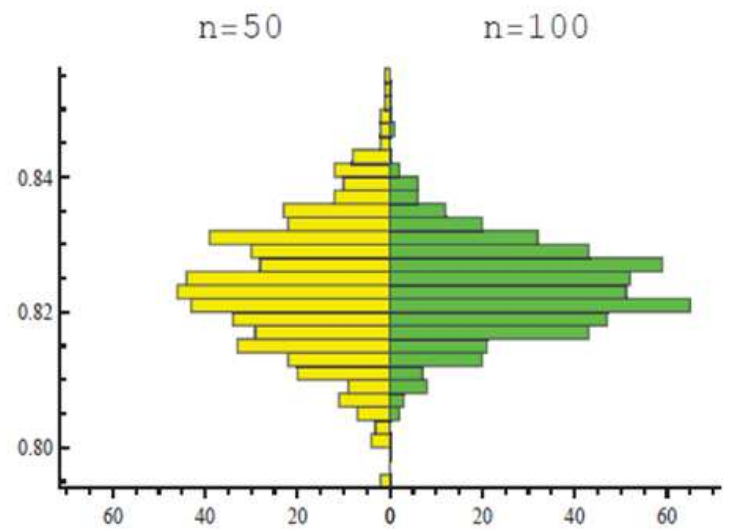

Variance

Variance

$\alpha=10, \beta=5, \lambda=1$

$$
\mathrm{n}=50 \quad \mathrm{n}=100
$$

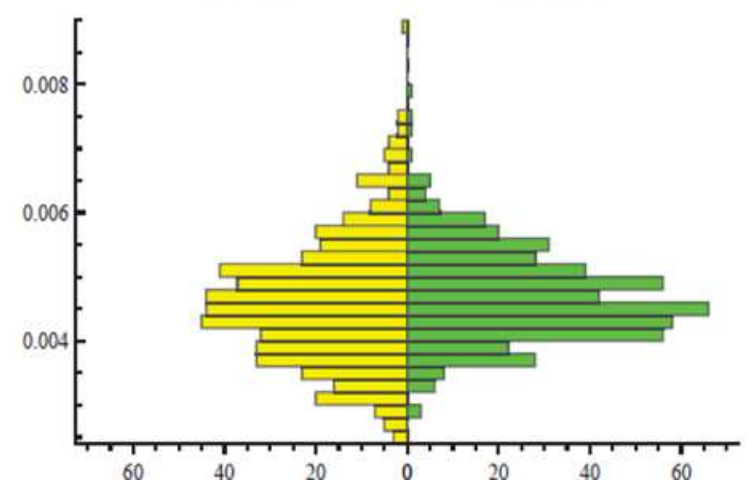

Coefficient of variation

$$
\alpha=10, \beta=5, \lambda=1
$$

$$
\mathrm{n}=50
$$

$\mathrm{n}=100$

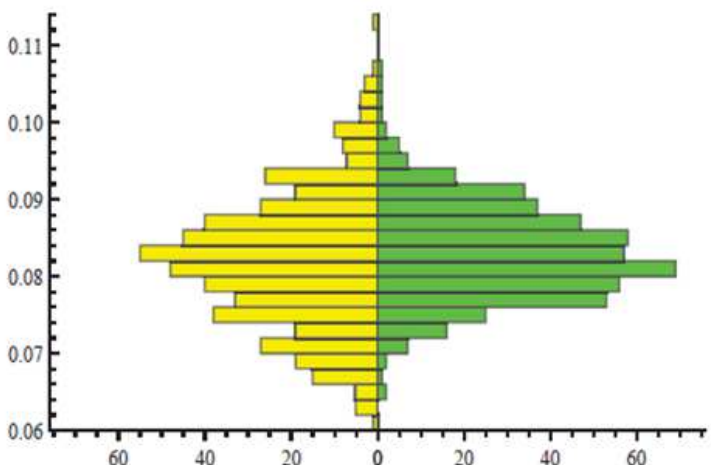

Fig. 4. Histograms of the sampling distributions of the MLEs of the mean, variance and coefficient of variation 


$$
\begin{gathered}
\text { Critical point of PDF } \\
\qquad \alpha=5, \beta=10, \lambda=1
\end{gathered}
$$

$\mathrm{n}=50$

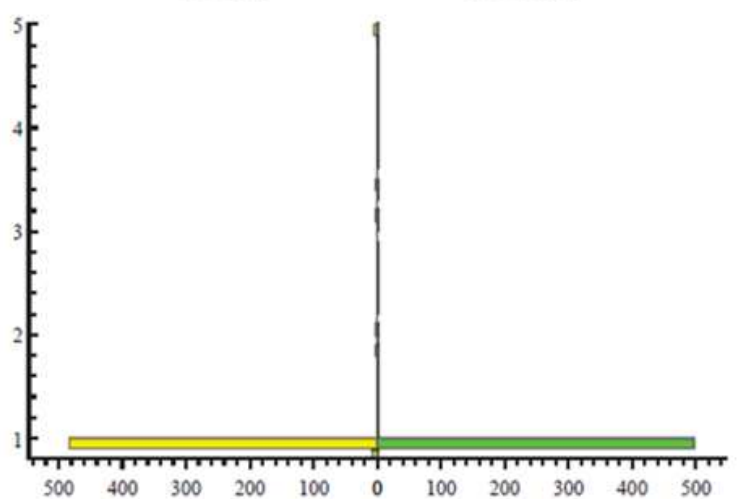

Critical point of PDF
\[ \alpha=10, \beta=5, \lambda=1 \]

$\mathrm{n}=50$

$\mathrm{n}=100$

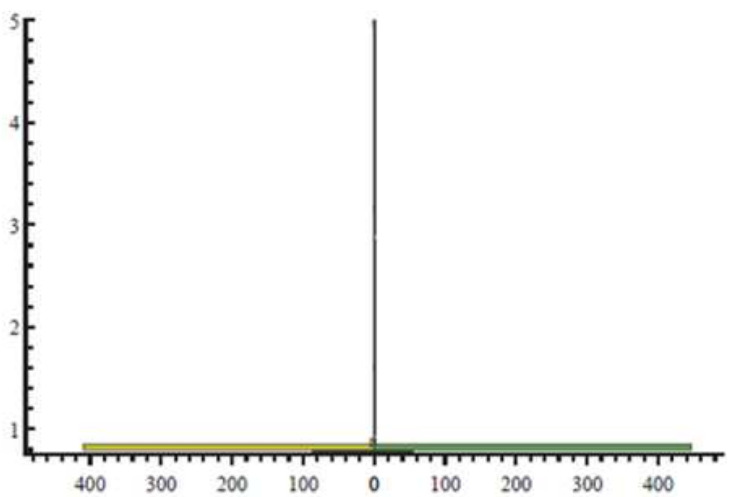

\section{Critical point of HRF $\alpha=5, \beta=10, \lambda=1$}

$$
\mathrm{n}=50 \quad \mathrm{n}=100
$$

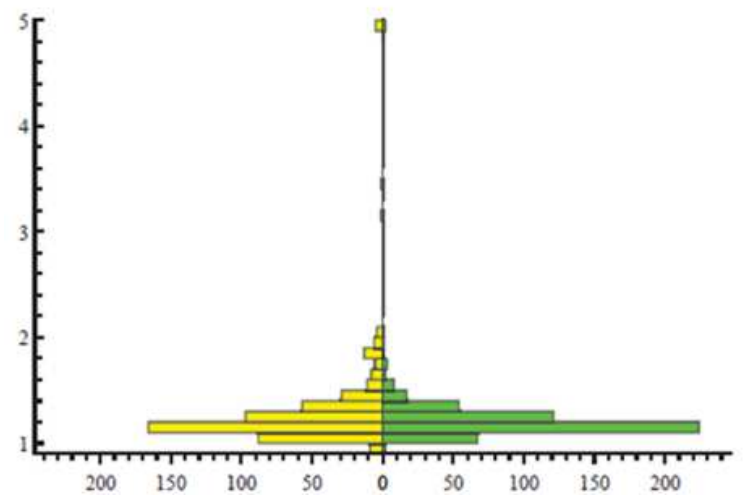

Critical point of MRLF

$$
\alpha=5, \beta=10, \lambda=1
$$

$$
\mathrm{n}=50
$$

$\mathrm{n}=100$

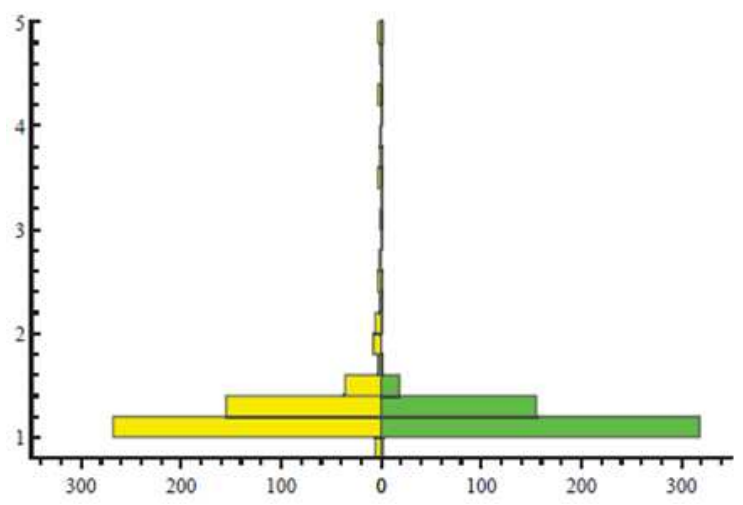

Critical point of $\mathrm{HRF}$

$$
\alpha=10, \beta=5, \lambda=1
$$

$$
\mathrm{n}=50
$$

$\mathrm{n}=100$

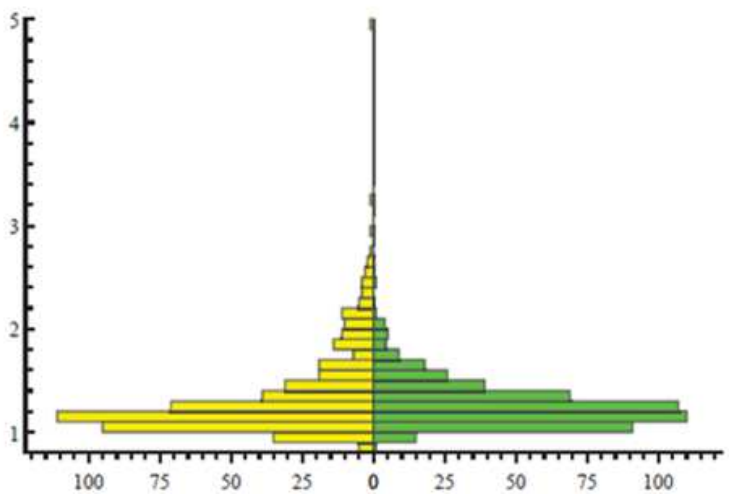

\section{Critical point of MRLF}

$$
\alpha=5, \beta=10, \lambda=1
$$

$\mathrm{n}=50$

$\mathrm{n}=100$

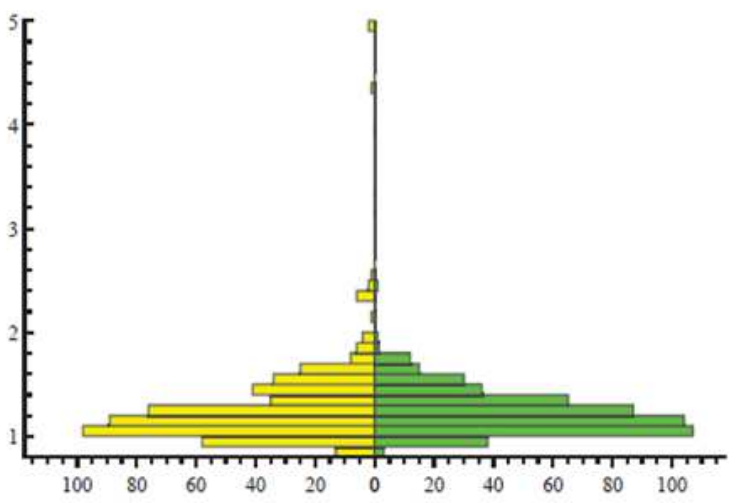

Fig. 5. Histograms of the sampling distributions of the MLEs of the critical point of PDF, HRF and MRLF 
Suja M. Aboukhamseen et al. / Journal of Mathematics and Statistics 2016, 12 (2): 86.98 DOI: $10.3844 / j m s s p .2016 .86 .98$

Table 4. Descriptive sample statistics

\begin{tabular}{llll}
\hline Minimum & 1.678 & Mean & 2.358 \\
Maximum & 3.210 & Variance & 0.075 \\
Mode & 2.438 & CV & 0.116 \\
\hline
\end{tabular}

Table 5. Parameters estimates, estimated log-likelihood, Kolmogrov-Smirnov and Anderson-Darling tests

Estimated Parameters

\begin{tabular}{lllllllr}
$\hat{\alpha}$ & $\hat{\beta}$ & $\hat{\lambda}$ & $\begin{array}{l}\text { Estimated } \\
\text { log-likelihood }\end{array}$ & KS & $p$-value & AD & $p$-value \\
\hline 16.391 & 3.098 & 44.632 & -10.425 & 0.082 & 0.531 & 0.385 & 0.863 \\
\hline
\end{tabular}

Table 6. Point and interval estimation for the mean, variance, coefficient of variation, critical points of PDF, HRF and MRLF Asymptotic Percentile bootstrap

\begin{tabular}{lllllllll}
$\theta$ & Estimate & S.E. & $95 \%$ C.I. & C.I. width & Estimate & S.E. & $95 \%$ C.I. & C.I. width \\
\hline$\mu$ & 2.358 & $0.028 *$ & $(2.304,2.412)$ & 0.108 & 2.357 & 0.028 & $(2.302,2.412)$ & 0.110 \\
$\sigma^{2}$ & 0.073 & $0.012 *$ & $(0.049,0.097)$ & 0.048 & 0.073 & 0.012 & $(0.052,0.099)$ & 0.047 \\
$c v$ & 0.115 & $0.009 *$ & $(0.096,0.134)$ & 0.038 & 0.114 & 0.009 & $(0.096,0.132)$ & 0.036 \\
$x_{f}$ & 2.321 & $0.039 *$ & $(2.244,2.398)$ & 0.154 & 2.322 & 0.039 & $(2.244,2.395)$ & 0.151 \\
$x_{h}$ & 4.045 & $0.913 *$ & $(2.255,5.835)$ & 3.580 & 4.258 & 0.913 & $(3.089,6.650)$ & 3.561 \\
$x_{m}$ & 3.945 & $0.950 *$ & $(2.083,5.807)$ & 3.724 & 4.160 & 0.950 & $(2.937,6.621)$ & 3.684 \\
\hline
\end{tabular}

*: bootstrap standard error

Empirical and theoretical PDFs

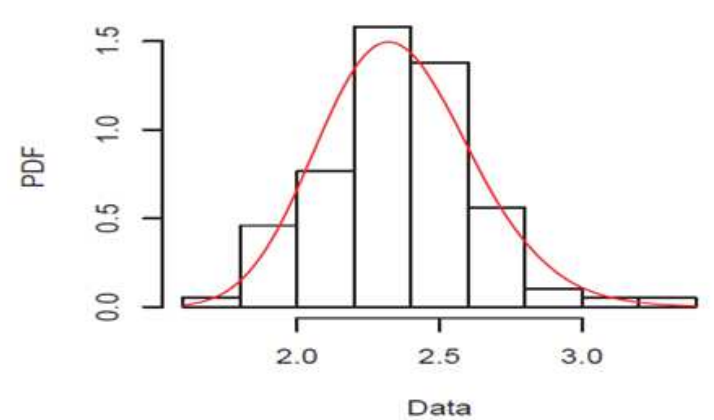

Empirical and theoretical CDFs

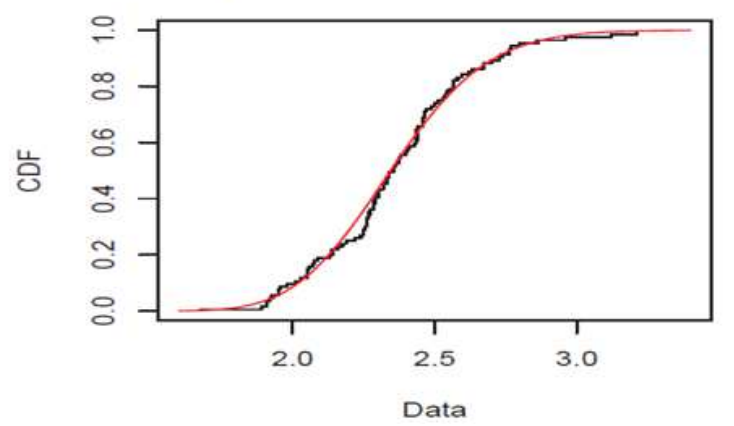

Q-Q plot

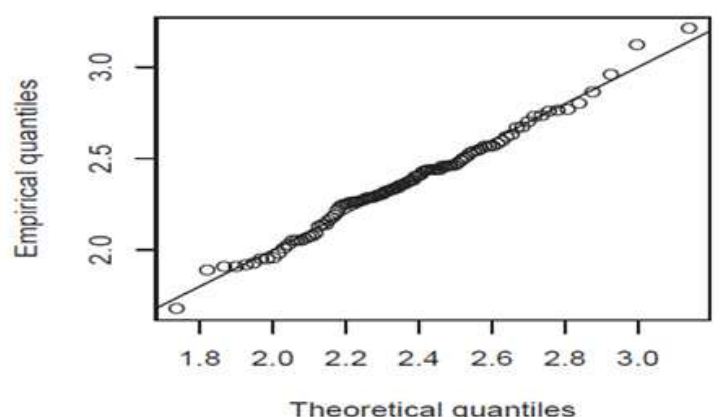

P-P plot

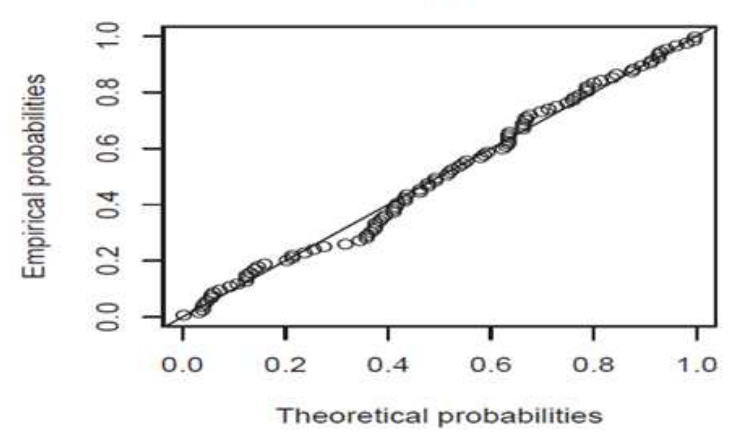

Fig. 6. Diagnostic plots for the fitted PHIW model

Finally, Table 6 compares the estimates of the considered functions of the parameters, their standard error and the corresponding confidence intervals based of asymptotic and percentile bootstrap estimation procedures. 

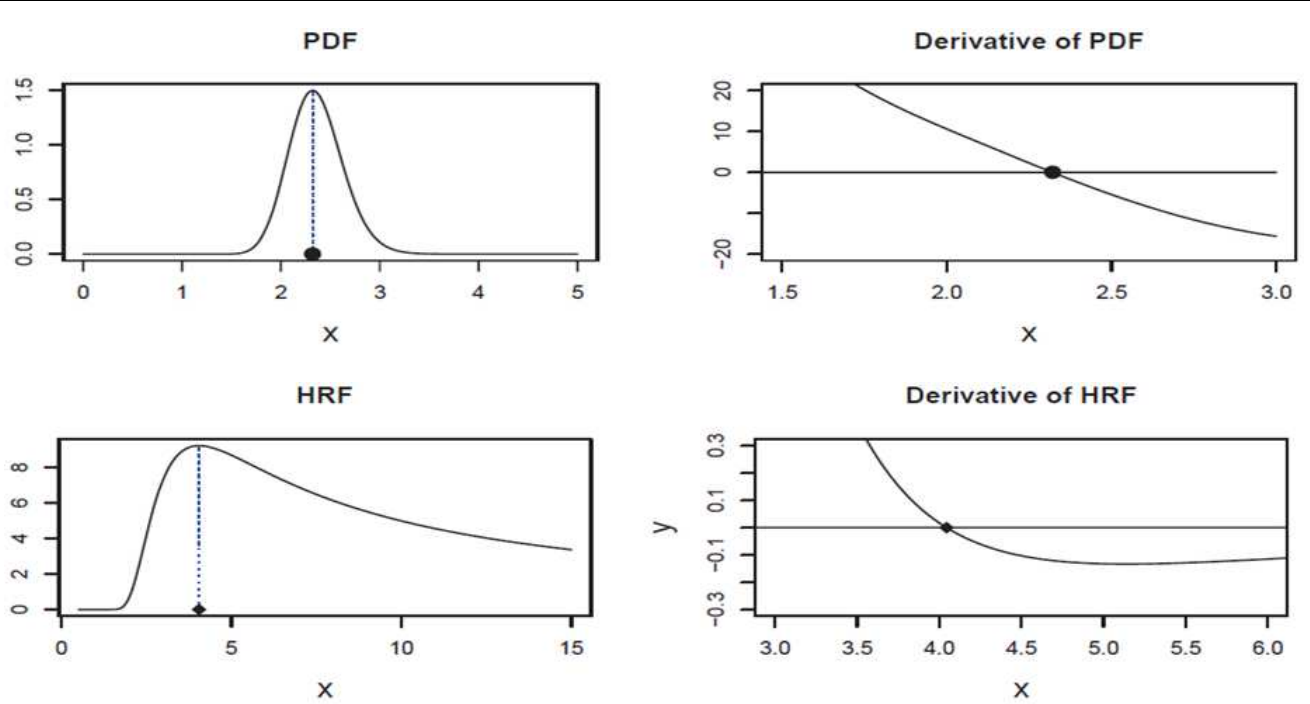

MRLF

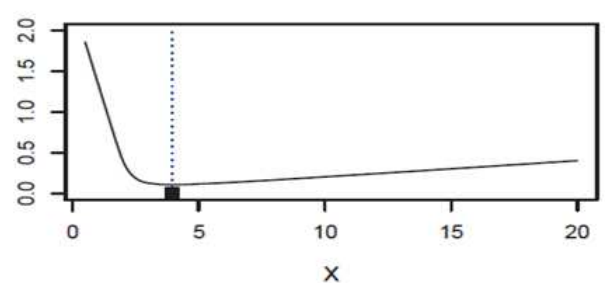

Derivative of MRLF

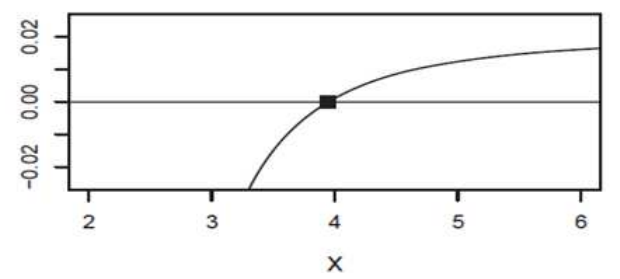

Fig. 7. Location of the percentile bootstrap estimates of the critical points of the PDF, HRF and MRLF of the fitted PHIW model
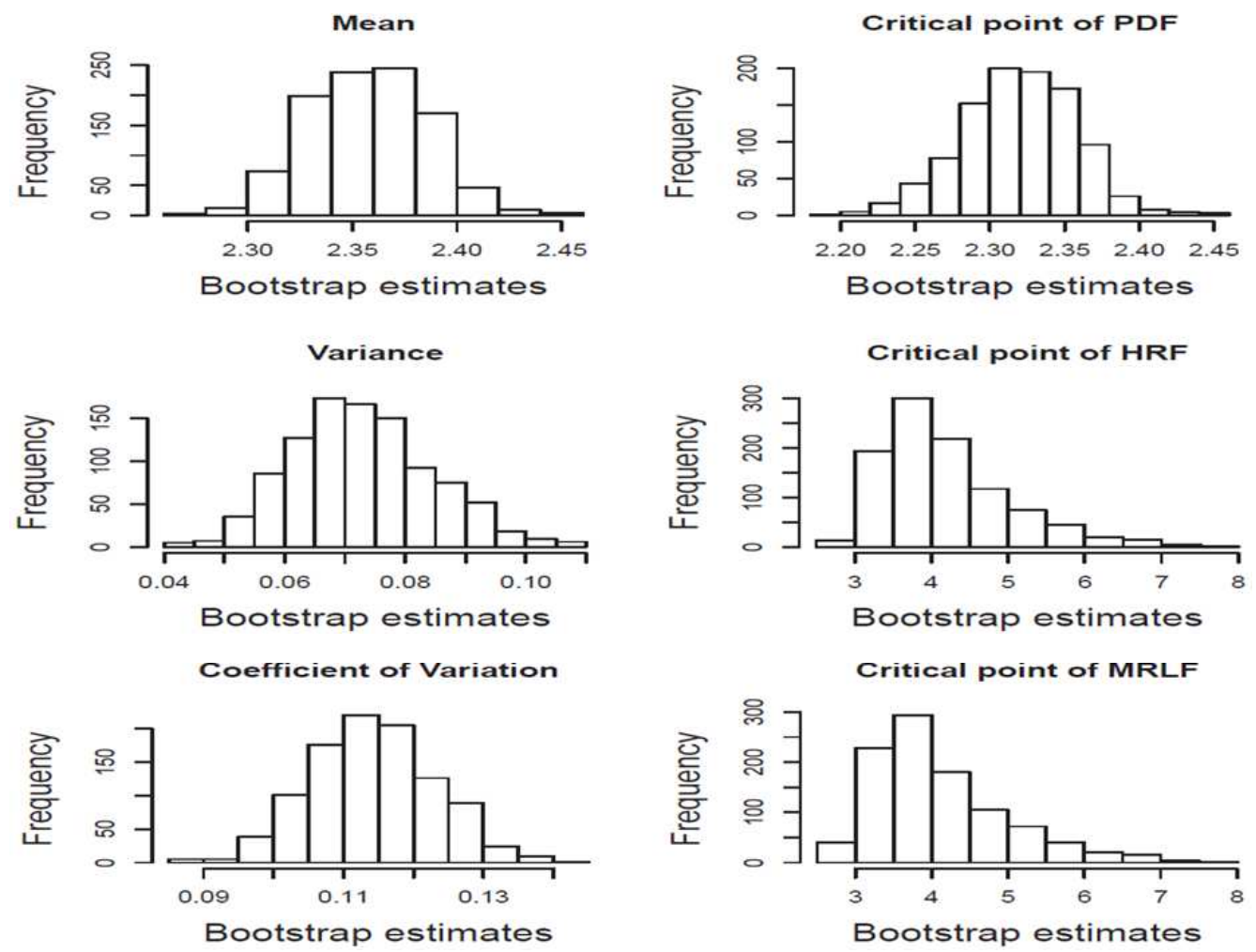

Fig. 8. Histograms of the percentile bootstrap estimates of the mean, variance, coefficient of variation (left column) and critical points of PDF, HRF and MRLF (right column) of fitted PHIW model 


\section{Conclusion and Comments}

In this study, we have studied the proportional hazard inverse Weibull distribution and the maximum likelihood/bootstrap estimation of various functions of its parameters. It has been shown that the density and hazard rate functions of the proposed model are unimodal for all values of the parameters and its mean residual life function is bathtub shaped under certain condition on two of its parameters. The simulation studies indicate that the asymptotic and bootstrap confidence intervals of various functions of the parameters perform similarly in terms of the coverage probabilities as well as the length of the confidence intervals. We hope that our investigation will provide an alternative way to analyze data which exhibit the characteristics similar to the proposed model.

\section{Author's Contributions}

All authors have contributed equally to the work.

\section{Ethics}

The authors declare that there is no conflict of interests regarding the publication of this article.

\section{References}

Abouammoh, A.M. and A.M. Alshingiti, 2009. Reliability estimation of generalized inverted exponential distribution. J. Stat. Comput. Simulat., 79: 1301-1315.

DOI: $10.1080 / 00949650802261095$

Calabria, R. and G. Pulcini, 1990. On the maximum likelihood and least squares estimation in the inverse Weibull distribution. Stat. Applic., 2: 53-66.

Canty, A. and B.D. Ripley, 2015. Boot: Bootstrap R (Splus) functions. R package version 1.3-15.

Choudhury, A., 2005. A simple derivation of moments of the exponentiated Weibull distribution. Metrika, 62: 17-22. DOI: $10.1007 / \mathrm{s} 001840400351$

Davison, A.C. and D.V. Hinkley, 1997. Bootstrap Methods and Their Application. 1st Edn., Cambridge University Press, Cambridge, ISBN-10: 0521574714, pp: 582.
Glaser, R.E., 1980. Bathtub and related failure rate characterizations. J. Am. Stat. Assoc., 75: 667-672. DOI: $10.1080 / 01621459.1980 .10477530$

Gupta, R.C. and O. Akman, 1995. Mean residual life function for certain types of non-monotonic ageing. Commun. Stat. Stochastic Models, 11: 219-225. DOI: $10.1080 / 15326349508807340$.

Gupta, R.C. and R.D. Gupta, 2007. Proportional reversed hazard rate model and its applications. J. Stat. Plann. Inference, 137: 3525-3536. DOI: $10.1016 /$ j.jspi.2007.03.029

Jiang, R., D.N.P. Murthy and P. Ji, 2001. Models involving two inverse Weibull distributions. Reliability Eng. Syst. Safety, 11: 219-225. DOI: $10.1016 / \mathrm{S} 0951-8320(01) 00030-8$

Khan, M.S., G.R. Pasha and A.H. Pasha, 2008a. Theoretical analysis of inverse Weibull distribution. WSEAS Trans. Math., 7: 30-38.

Khan, M.S., G.R. Pasha and A.H. Pasha, 2008b. Fisher information matrix for the inverse Weibull distribution. Int. J. Math. Sci. Eng. Applic., 2: 257-262.

Lehmann, L.E. and G. Casella, 1998. Theory of Point Estimation. 2nd Edn., Springer, New York.

Marshall, A.W. and I. Olkin, 2007. Life Distributions: Structure of Nonparametric, Semiparametric and Parametric Families. 1st Edn., Springer, New York, ISBN-10: 0387684778, pp: 788.

Mudholkar, G.S. and D.K. Srivastava, 1993. Exponentiated Weibull family for analyzing bathtub failure-rate data. IEEE Trans. Reliability, 42: 299-302. DOI: $10.1109 / 24.229504$

RDCT, 2014. R: A language and environment for statistical computing. R Foundation for Statistical Computing, Vienna, Austria.

Shao, Q., H., Wong, J. Xia and W.C. Ip, 2004. Models for extremes using the extended three-parameter Burr XII system with application to flood frequency analysis. Hydrol. Sci. J., 49: 685-702. DOI: $10.1623 /$ hysj.49.4.685.54425 